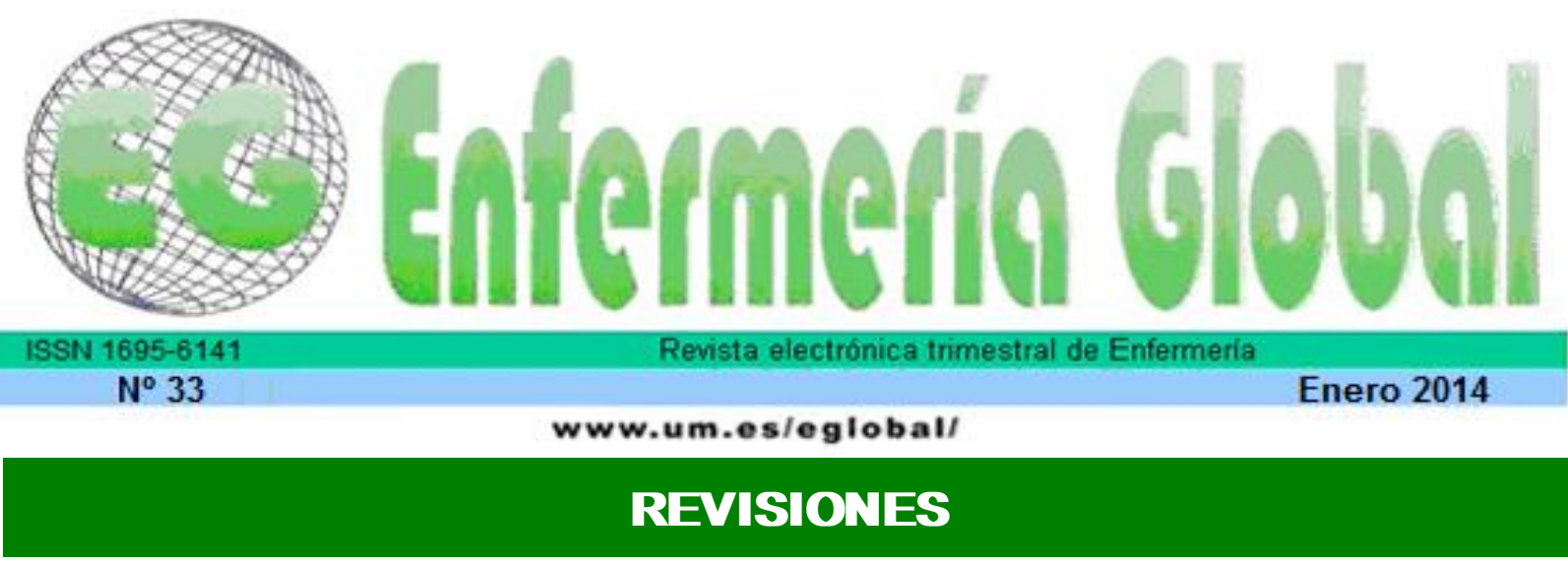

\title{
Delirium postoperatorio en cirugía general, el fantasma de nuestros abuelos
}

Postoperative delirium in general surgery, the ghost of our grandparents

\section{${ }^{*}$ Carrera Castro, Carmen}

*Enfermera. Hospital Ernest LLuch. Calatayud. Zaragoza E-mail:ccarrera@salud.aragon.es

Palabras clave: delirium; anciano; cirugía; factores de riesgo; postoperatorio.

Keywords: labour; pain; analgesic alternatives.

\section{RESUMEN}

En esta revisión descriptiva se realiza una búsqueda bibliográfica en las principales bases de datos biomédicas para detallar los factores de riesgo modificables vinculados al delirium postoperatorio en pacientes ancianos, intervenidos de cirugía general, con la finalidad de optimizar la calidad asistencial.

Se obtuvieron siete estudios observacionales que describen los factores de riesgo y predictores independientes de riesgo asociados al delirium postoperatorio.

Los factores de riesgo modificables que precipitan este síndrome, en este subgrupo de pacientes quirúrgicos, y que los hacen característico en relación a los pacientes médicos, son el tiempo de ingreso en relación con el procedimiento quirúrgico, la duración de la cirugía, la estancia prolongada en unidad de cuidados intensivos, la hipotensión intraoperatoria, el volumen de infusión de líquidos elevados, la puntuación ASA (Sociedad Americana de Anestesiólogos) alta, saturación de oxigeno cerebral disminuida, dolor postoperatorio mal controlado, retraso en la recuperación de la anestesia y mayor numero de complicaciones postoperatorias.

Los ancianos intervenidos de cirugía general son un grupo de pacientes con alto riesgo de delirium postoperatorio debido al gran número de factores de riesgo adicionales anexados a su proceso quirúrgico.

La identificación de pacientes en riesgo es fundamental para la prevención, la detección temprana y el tratamiento adecuado del delirium, primordialmente porque el delirium indica la presencia de una alteración orgánica subyacente que debe resolverse.

\section{ABSTRACT}

Introduction: Currently there has been an increased interest from mothers and their environment, by the application of other non-invasive analgesic methods. Therefore, it is a major issue for health professionals related to delivery care, which should develop the skills necessary to help those women who choose childbirth alternative promoted by the application of analgesic action.

Objectives: We sought to evaluate the effectiveness and efficiency of the main alternatives analgesic delivery that literature offers us. 
Methods: We performed a literature search in the following databases: Medline, Pubmed, The Cochrane Database of Systematic Reviews and Ebsco.

Results: The studies surveyed reflect that different alternatives as applying analgesic electrotherapy, acupuncture, massage therapy or thermotherapy in childbirth are moderate effective in reducing pain.

Conclusions: The application of alternative analgesic action is proven effective, however, it is necessary the development of larger clinical studies with a methodology agreed to endorse the effectiveness of such interventions.

\section{INTRODUCCIÓN}

Cada vez más, somos más longevos y con mayor necesidad de intervención quirúrgica ${ }^{1,2}$

Antes la edad, per se, era un impedimento ${ }^{3}$ a la hora de decidir si se intervenía o no, pero actualmente no es asi ${ }^{2}$, bien sea por las mejoras tecnológicas y la mejor calidad de vida o simplemente porque la población presente y futura es cada día más octogenaria.

Los abuelos, a diferencia de otros grupos mas jóvenes, sufren una situación biológica normal de envejecimiento con el consiguiente descenso gradual e irreversible de sus funciones biológicas y capacidad funcional, esto unido a la hospitalización, escaso apoyo familiar o social, la pluripatología y la polifarmacia, les hacen más vulnerables y frágil ante el estrés quirúrgico, de padecer delirum postoperatorio ${ }^{4-6}$; siendo el delirium una complicación frecuente y perjudicial entre los ancianos operados ${ }^{1,7,8}$, además es un síntoma psicogeriatrico de emergencia médica ${ }^{9}$.

El Delirium es considerado un Síndrome cerebral, grave, orgánico, de inicio súbito, multifactorial $^{10}$, transitorio, potencialmente predecible, prevenible y reversible ${ }^{3,5-6,11-17}$, donde la enfermería juega un papel primordial a la hora de aplicar intervenciones proactivas multifactoriales, protectoras ante los factores de riesgo modificables, con la finalidad de prevenir o minimizar los efectos del delirium, gracias al constante y habitual apoyo psicológico, proximidad y cuidados que les brindamos al paciente ${ }^{18}$.

\section{EI Postoperatorio Quirúrgico. Tipos de Delirium.}

El período postoperatorio comienza inmediatamente después de la cirugía y continúa hasta que el paciente es dado de alta. Se divide en el preoperatorio inmediato (dura aproximadamente 2-4 horas.) y el tardío (desde su llegada a planta de hospitalización hasta su alta hospitalaria). ${ }^{19}$

Durante el periodo del postoperatorio se pueden dar dos tipos ${ }^{20}$ de deterioros cognitivos: El delirium postoperatorio (DPO) y la disfunción cognitiva postoperatoria (DCP). Esta revisión centrará su temática en el delirium postoperatorio.

El concepto de Disfunción cognitiva postoperatoria (DCP) genera controversias y existen diferentes definiciones en la literatura médica ${ }^{20-21}$, se podría especificar como una disfunción compleja y multifactorial, que se caracteriza por alteraciones en la función cognitiva generando alteraciones en la memoria, concentración, procesos de información e integración social, relacionado con un acontecimiento quirúrgico. 
El Delirium, también llamado Síndrome Confusional Agudo (SCA), se puede definir según el manual diagnóstico y estadístico de los trastornos mentales (DSM-IV-TR2) como resultado de la suma de "una alteración del nivel de conciencia con disminución de la capacidad para centrar, mantener o dirigir la atención unido a un cambio en las funciones cognoscitivas o presencia de una alteración perceptiva que no se explica por la existencia de una demencia previa o en desarrollo, pudiéndose presentar en un periodo corto de tiempo, días o horas, y fluctuar a lo largo del día".

En base a la alteración del nivel de conciencia y actividad psicomotriz ${ }^{23-24}$ el delirium se clasifica en 4 subtipos: Hiperactivo, Hipoactivo, Mixto y sin Actividad psicomotriz. El Hipoactivo es el más frecuente e infradiagnosticado a causa de su presentación clínica, con alto riesgo de úlceras por presión y peor pronóstico tras el postoperatorio $^{25}$.

Dentro del periodo postoperatorio nos podemos encontrar lo que sería el delirum emergente (postoperatorio inmediato) que aparece tras revertir la anestesia general, suele durar minutos a horas, y el delirium, propiamente dicho, postoperatorio, (postoperatorio tardío) que trascurre a partir del tercer día de la intervención quirúrgica, con un intervalo previo de días con lucidez y se revierte en horas a días, aunque puede llegar a durar semanas a meses ${ }^{26}$.

\section{Delirium postquirúrgico en el Anciano.}

La incidencia de este síndrome en pacientes ancianos es elevada y variable dependiendo de la población a estudio, tipo de intervención (grado de estrés operatorio), métodos de detección y criterios diagnósticos ${ }^{5,27-28}$. En correlación al tipo de intervención quirúrgica, la incidencia del delirium en cirugía electiva produce menor riesgo de padecer delirium que la de urgencia; la cirugía ortopédica y en concreto la fractura de cadera son las que se presentan con rangos de incidencia mayores (35\%$65 \%$ ), en la cirugía cardiaca se da en segundo lugar de frecuencia, con valores variables dependiendo del tipo de intervención (aneurisma de aorta abdominal entre $33 \%-54 \%$, cirugía de revascularización coronaria de injerto 37\%-52\%, etc.), quedando en un tercer lugar la cirugía abdominal, que oscila entre 5\%-51\% de incidencia de delirium DPO.

La etiología es multifactorial, resulta de la interacción entre la vulnerabilidad previa del anciano y los factores precipitantes del entorno quirúrgico, lo que se traduce en un anarquía celebral ${ }^{10}$; la fisiopatología (figura 1.) sigue mostrado grietas en su esclarecimiento, se debate por dilucidar diferentes teorías como la de la inflamación ${ }^{29}$, los neurotransmisores ${ }^{29}$, el estrés ${ }^{29}$ y reservas cognitivas disminuida ${ }^{30}$ pero la hipótesis con mayor peso es la de la "Neurotransmisión", donde se produce una hipofunción colinérgica con un aumento conjunta de la liberación de la dopamina y serotonina, consecuencia de acto operatorio, dando lugar una disminución de la actividad cerebral ${ }^{31}$. 


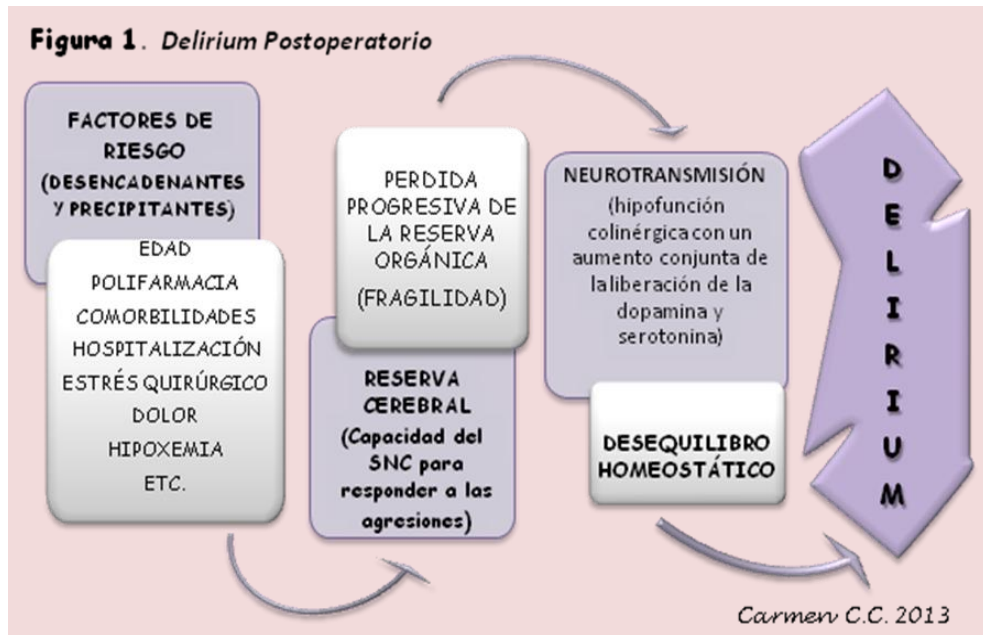

Los síntomas prodrómicos característicos de estos pacientes son la desorientación y las llamadas urgentes para ser atendidos ${ }^{32}$, los cuales se enfatizan más durante el periodo nocturno, dando lugar a los trastornos de ciclo sueño-vigilia ${ }^{33}$.

Lo evidente, es que tenemos instrumentos para la detección precoz del DPO, a partir de los cuales podemos identificar a los pacientes en riesgo, escalas para diagnosticarlos en su fase inicial, cuantificar su gravedad y realizar un seguimiento de su evolución ${ }^{34}$. Un ejemplo de estos instrumentos sería la escala para el diagnóstico: "Confusion Assessment Method" (CAM) ${ }^{35}$, se realiza en unos cinco minutos aproximadamente, tiene una sensibilidad del $94-100 \%$ y especificidad del $90-95 \%$. Debemos de tener muy presente que cuando el delirium se instaura es más difícil revertirlo y en estados de agitación corremos riegos todos ${ }^{36}$.

El riesgo de DPO es la suma de los factores de riegos predisponentes y precipitantes ${ }^{9}$.

Se han ido creando distintos modelos de predicción del DPO en grupos diferentes de pacientes quirúrgicos, con heterogeneicidad de sus factores, lo que problematiza la totalidad de un modelo unificado, un ejemplo de estos predictores para el grupo de pacientes no cardiacos sería: Fármacos preoperatorios, consumo de alcohol y tabaco, historia de enfermedades agudas o crónicas, enfermedades neurológicas o psicológicas y historia de delirium previo. ${ }^{37}$

Los pacientes médicos (no quirúrgicos), a diferencia de los quirúrgicos, no sufren el estrés operatorio con la consiguiente respuesta inflamatoria sistémica, ni están sometidos a la anestesia, la hipotermia y el manejo del dolor postoperatorio, los cuales son algunos factores de riesgo potencialmente añadidos al DPO. ${ }^{9}$

\section{La Magnitud del Delirium en sus Tres Planos}

Para el sistema sanitario es un problema importante de salud pública, de tal relevancia que el diagnóstico de delirium ha sido incorporado dentro de los indicadores de calidad de agencias internacionales como la americana "Agency for Healthcare Research and Quality" ${ }^{\prime 38}$. Se da con alta frecuencia a todos los niveles asistenciales, produce elevado costo sanitario y sobrecarga laboral para el personal sanitario, en ocasiones, son los propios servicios de salud los que contribuimos a su desarrollo y agravamiento de forma indirecta ${ }^{33,39-42}$. 
En el ámbito hospitalario sigue estando mal diagnosticado y tratado ${ }^{39,42-43}$ a nivel postoperatorio no se reconoce ni trata hasta un $80 \%$ de los pacientes. ${ }^{44}$

Existen autores que informan sobre el manejo empírico del delirium, sin utilizar guías basadas en la evidencia científica sobre el diagnóstico y la gestión. ${ }^{45}$

Resulta ser el segundo síndrome psiquiátrico más usual hospitalario, después de los trastornos depresivos. ${ }^{46}$

Para el paciente supone una asociación de mayor morbilidad, mortalidad, deterioro funcional persistente, estancia hospitalaria, rehospitalización, rehabilitación, institucionalización comunitarias, con peor evolución y pronóstico, además de ser un precursor de la demencia. ${ }^{39,41-42,44}$

El Delirium interfiere, en ocasiones, sobre los cuidados específicos del paciente, como la administración de medicamentos, el tratamiento de heridas, fisioterapia, alimentación, higiene y planificación del alta. ${ }^{39}$

Origina un aumento del riesgo de desarrollar complicaciones intrahospitalarias, como las infecciones nosocomiales, úlceras por presión, incontinencias y caídas accidentales. $^{47}$

A pesar de ser un síndrome agudo y potencialmente reversible existen ancianos que tras el alta hospitalaria continúan padeciendo sus efectos ${ }^{33}$, e incluso no desaparecer completamente y pueden quedarse con un grado de incapacidad permanente. ${ }^{48}$

Para la familia o cuidador principal y el personal sanitario genera una mayor inseguridad física y psíquica, con repercusiones negativas sobre la autoestima profesional. ${ }^{36,49-50}$

Genera un sentimiento degradante, angustioso y frustrante en el anciano ante una situación en la que pierde el control de si mismo, unido a la ansiedad y sufrimiento del familiar o cuidador principal. ${ }^{16,39,50}$

Todo esto se traduce en un deterioro personal (paciente), familiar, profesional, social y sanitario. $^{42}$ Aunque es un marcador de la calidad de la atención hospitalaria ${ }^{38,51}$ su incidencia y consecuencias negativas son elevadas. ${ }^{5,27-28}$

\section{El Abordaje Terapéutico}

Las intervenciones proactivas para la prevención y tratamiento no farmacológico de delirum van encaminadas al control de los factores de riesgo modificables, la valoración de los factores predisponentes, el tratamiento específico de las causas subyacentes, alteraciones del comportamiento y cambios en el medio ambiente, al igual que deberemos estar pendiente de prevenir las potenciales complicaciones derivadas del estatus confusional. ${ }^{8,17,39,44}$

El manejo farmacológico del delirium está justificado para los pacientes que tras el uso de medidas no farmacológicas no han logrado la remisión del cuadro confusional $^{9}$, aunque su eficacia no está claramente demostrada. ${ }^{17}$ 
La identificación de los factores de riesgo, el diagnóstico precoz y el tratamiento en fase inicial, pueden reducir la incidencia y consecuencias de delirium, además del costo hospitalario. ${ }^{9,52}$

La identificación especifica de los factores de riesgo modificables del delirium en el postoperatorio por el personal sanitario es una intervención transcendental porque supone la base del éxito tanto para las estrategias de prevención como de tratamiento, no farmacológicas, en fase inicial, ya que son las más efectivas desde el punto de vista clínico, por encima de las farmacológicas, a la hora de prevenir el delirium $^{17}$, lo que se traduce en una mayor calidad y seguridad en la atención del anciano quirúrgicos. ${ }^{42,52}$

Todas las razones expuestas anteriormente justifican la redacción de esta revisión.

Esta revisión bibliográfica descriptiva tiene como objetivo detallar cuáles son factores de riesgo modificables que se encuentran vinculados al delirum durante el postoperatorio, en el colectivo de pacientes ancianos, intervenidos de cirugía general, durante la estancia hospitalaria, con la finalidad de optimizar la calidad asistencial mediante la prevención y el tratamiento no farmacológico y adquirir una visión actualizada de esta subpoblación de pacientes quirúrgicos poco analizada.

\section{METODOLOGÍA}

Este artículo de revisión bibliográfica descriptiva narrativa se inicia con una búsqueda selectiva bibliográfica en las principales bases de datos biomédicas, como: PubMed, Cochrane Plus, BVS, MEDES, el motores de búsqueda como: Google Académico. Se utilizó la combinación de operadores boléanos (AND, NOT, OR) con los descriptores anexado a la base de datos MeSH: "delirium", "anciano", "cirugía", "factores de riesgo" y "postoperatorio". A la vez se fue agrupando y examinando los estudios originales que se ajustaban al objetivo de la revisión.

Los criterios de selección son artículos originales publicados, sobre estudios observacionales, en idioma inglés o español, de libre difusión o en su defecto el resumen, que compendre información sobre la asociación de los factores de riesgo y el delirium postoperatorio en Cirugía Mayor General, abierta o por laparoscopia, se excluyen trabajos realizados en unidad de cuidados intensivos y estudios con heterogeneicidad en poblaciones de pacientes quirúrgicos que no sean de Cirugía General. En Humanos Ancianos, en ambos sexos. Periodo de búsqueda se acotó entre diciembre del 2007 diciembre del 2012.

En PubMed se utilizo la siguiente estrategia de búsqueda: (("delirium"[MeSH Terms] OR "delirium"[All Fields]) AND ("aged"[MeSH Terms] OR "aged"[All Fields] OR "elderly"[All Fields]) AND ("surgical procedures, operative"[MeSH Terms] OR ("surgical"[All Fields] AND "procedures"[All Fields] AND "operative"[All Fields]) OR "operative surgical procedures"[All Fields] OR "surgical"[All Fields]) AND ("patients"[MeSH Terms] OR "patients"[All Fields])) AND ("2007/12/16"[PDat] : "2012/12/13"[PDat] AND "humans"[MeSH Terms]), se obtuvo 281articulos de los que seleccionó 5 artículos que se regía a los criterios que forman el objetivo de este trabajo.

En "PubMed Clinical Queries" se creó un batida con la palabra "delirium", resultando fallida la indagación, al igual que la búsqueda en Cochrane Plus y en MEDES. 
En BVS se utilizó la combinación de los descriptores en inglés: "delirium AND risk factors AND surgery", acotando la búsqueda al grupo de ancianos, últimos 5 años e idioma en inglés, se obtuvo 52 artículos de los cuales 1 se ajustaba a los criterios de selección, pero había sido seleccionado previamente en la búsqueda de PubMed; con los descriptores en castellano "delirium AND cirugía", limitando al grupo de ancianos se obtuvieron 27 resultados, uno de los artículos fue aceptado.

En Google Académico se fue combinando las palabras en inglés de: delirium, factores de Riesgo, ancianos y cirugía; se limitó a 2007-2012, se obtuvieron 119 referencias bibliográficas, obteniendo 1 estudio válido y no repetido.

Los resultados obtenidos en la búsqueda fueron siete trabajos observacionales entre descriptivos y analíticos.

\section{RESULTADOS}

Por su naturaleza multifactorial, el delirium se forma a partir de la sinergia entre los factores de riesgos inherentes a pacientes, los que marcan su vulnerabilidad y están ligado a la fragilidad de anciano, llamados Factores de Riesgo Predisponentes, y los Factores externos Precipitantes que son los coadyuvantes de la génesis de este síndrome. $^{47}$

A lo largo de la literatura médica nos encontramos diferentes trabajos actuales que nos hablan sobre los factores de Riesgo y predictores independientes de DPO en la subpoblación de ancianos quirúrgicos intervenidos de Cirugía General (Tabla I)

En 2007 se publicó un estudio retrospectivo de series de $\operatorname{casos}^{53}$ con 228 pacientes, mayores de 70 años, intervenidos en cirugía abdominal, 89 casos se identificaron con alto riesgo de desarrollar DPO basándose en la presencia de al menos tres de los siguientes factores de riesgo: enfermedad grave, discapacidad visual, deterioro cognitivo y la deshidratación. Los resultados del estudio mostraron que el estado nutricional y el deterioro funcional preoperatorio son importantes predictores independientes de delirio postoperatorio. Se dio una diferencia pequeña, pero estadísticamente significativa, en la edad, los que desarrollaron delirio, en promedio, fueron cuatro años mayores (81 años, 95\% Cl, 79-82 años) versus aquellos que no (77 años, 95\% Cl, 75-79 años, $P=$. 005). La incidencia de DPO fue elevada (60\%) los autores explican que puede estar relacionado con una "atención inadecuada" en áreas previamente asociados con el delirio. Los factores de riesgo clínicos presumiblemente asociados con el delirum en el postoperatorio descrito por estos autores fueron: Restricción física, cateterismo vesical, administración de más de cuatro medicamentos nuevos, uso de medicamentos precipitantes, reposo en cama prolongado, estado pobre en fluidos, mal control glucémico, hipoxia, anemia, dolor no controlado, trastornos metabólicos, hipoalbuminemia, malnutrición, morbilidad y falta de precaución en las caídas.

Koebrugge $\mathrm{B}^{54}$ y colaboradores realizaron un estudio Observacional descriptivo prospectivo, publicado en el año 2009, con una población reducida de 71 pacientes de 65 años o más, sometidos a cirugía abdominal electiva. En el análisis univariado expuso como factores de riesgo importantes para el delirio postoperatorio: mayores de 74 años, estancias prolongadas en UCl (Unidad de cuidados intensivos) y hospitalarias, mayor número de complicaciones postoperatorias, las bajas puntuaciones cognitivas preoperatorio en las pruebas de detección, y la puntuación 
intraoperatoria de ASA (Sociedad Americana de Anestesiólogos) elevada. Tras la realización del análisis multivariado sólo la edad avanzada de 74 años o más (OR 1.16; 95\% Cl 1.00-1.35; $p=0.05$ ) fue un factor de riesgo significativo. La incidencia de DPO fue del 24\%. Destacar de este estudio que los investigadores no controlaron el uso de medicamentos y la muestra a estudio fue pequeña.

Otro estudio Observacional prospectivo ejecutado por Morimoto $\mathrm{Y}$ et al. ${ }^{55}$ descubrieron que la edad avanzad (74+/-4 años), la función cognitiva preoperatoria baja, la saturación de oxígeno cerebral preoperatoria disminuida y el retraso en la recuperación de la anestesia son factores de riesgo del DPO en pacientes sometidos a cirugía abdominal electiva, pero concluyen resaltando los tres primeros factores de riesgo como estadísticamente significativos. El estudio fue desarrollado con una pequeña muestra de 20 pacientes mayores de 65 años, tras excluirse a 3 pacientes con patología cerebral o demencia de base. En el postoperatorio, 5 (25\%) de los pacientes tuvieron delirium. Reseñar que la saturación de oxígeno cerebral no se vigiló después de la cirugía, así que los posibles déficits postoperatorios no se han tenido en cuenta.

Tei $\mathrm{M}$ et al. ${ }^{56}$ realizaron un estudio con 129 pacientes mayores de 71 años con cáncer colorrectal asistidos por laparotomía (81 pacientes) o laparoscopia (48 pacientes). En este estudio Observacional retrospectivo la edad, la puntuación del ASA y PNI (índice de pronóstico nutricional) en los pacientes con DPO fue significativamente mayor, a diferencias de los que no lo tenían ( $p<0,05$, cada uno). El análisis de regresión logística identificó PNI y la encefalopatía como dos factores de riesgo independientes para desarrollar el DPO. Se diagnosticó una incidencia del 10,9\% de DPO en estos pacientes.

En el 2010, Ansaoni L y colaboradores ${ }^{57}$ publicaron un estudio de caso-control con 351 pacientes mayores de 65 años procedentes de cirugía general electiva y de urgencias, asociaron la edad avanzada (>75 años), la presencia de co-morbilidades, el deterioro cognitivo preoperatorio, los síntomas psicopatológicos y mal control glucémico con el DPO. La incidencia del delirum fue 13,2\%, algo más elevada cuando la cirugía era de urgencia $(17,9 \%)$.

Patti $\mathrm{R}$ et al. ${ }^{58}$ en 2011 divulgaron un estudio observacional prospectivo donde se originó un $18 \%$ de DPO de los 100 pacientes mayores de 65 años sometidos a cirugía colorrectal por cáncer. En el análisis univariante relacionaron de forma importante a la edad avanzada, el abuso de alcohol, antecedentes previos de DPO, hipoalbuminemia, hipotensión intraoperatoria, el volumen de infusión elevado y la pérdida excesiva de sangre con el desarrollo del DPO, pero cuando se realiza el análisis multivariado solo da como factores de riesgo independientes de DPO la hipoalbuminemia, la hipotensión y el abuso de alcohol.

El último artículo que vamos a reflejar en esta revisión fue realizado por Mangnall LT y colegas $^{59}$, publicado recientemente, en 2011 , y con una población de 118 pacientes intervenidos de cirugía colorrectal, demostraron mediante un estudio observacional descriptivo prospectivo que los predictores independientes de DPO, analizados por regresión logística, eran la edad avanzada, no estar casado, ser hombre, admisión temprana (tiempo que transcurre desde la hospitalización y la cirugía) y la estancia de una noche en la UCl. La incidencia de DPO en las primeras 24 horas fue del $21 \%$ y a los tres días de $35 \%$, conforme avanzaba los días el porcentaje de nuevos casos disminuía. Destacar que al comparar los pacientes con delirium de los que no, los 
factores de riesgo como la edad, estar soltero, tener una deficiencia de movilidad y haber tenido un ingreso en la unidad de cuidados intensivos era más frecuente en pacientes con DPO.

I.Tabla I Predictores independientes y Factores de riesgo de Delirium en el postoperatorio tras Cirugía General en Ancianos.

\begin{tabular}{|c|c|c|c|c|}
\hline $\begin{array}{l}\text { AUTOR } \\
\text { ES } \\
\text { AÑOS }\end{array}$ & $\begin{array}{l}\text { POBLACIÓN } \\
\text { ESTUDIOS }\end{array}$ & $\begin{array}{l}\text { CIRUGÍA } \\
\text { GENERAL/ } \\
\text { INCIDENCIA }\end{array}$ & $\begin{array}{l}\text { FACTORES DE } \\
\text { RIESGO } \\
\text { POSTOPERATO } \\
\text { RIOS }\end{array}$ & $\begin{array}{l}\text { PREDICTORE } \\
\text { S } \\
\text { INDEPENDIE } \\
\text { NTES }\end{array}$ \\
\hline $\begin{array}{l}\text { Mangn } \\
\text { all LT } \\
\text { et al. }{ }^{59} \\
2011\end{array}$ & $\begin{array}{l}\mathbf{N}=\mathbf{1 1 8} \\
\text { pacientes con } \\
\text { cirugía } \\
\text { colorrectal } \\
\text { Edad Media } \\
71,81 \text { años } \\
\text { OBSERVACIO } \\
\text { NAL } \\
\text { DESCRIPTIVO } \\
\text { PROSPECTIVO }\end{array}$ & $\begin{array}{l}\text { CIRUGÍA } \\
\text { GASTRO- } \\
\text { INTESTINAL } \\
\text { COLORREC } \\
\text { TAL } \\
\text { DPO* } \\
24 h: 21 \% \\
\text { DPO }{ }^{*} 3^{\circ} \text { día: } \\
35 \%\end{array}$ & $\begin{array}{l}\text { Edad avanzada, } \\
\text { hombre, soltero, } \\
\text { limitaciones } \\
\text { físicas, } \\
\text { comorbilidades, } \\
\text { admisión en } \\
\text { UCI }{ }^{\dagger} \text {, admisión } \\
\text { temprana, } \\
\text { duración de la } \\
\text { cirugía, niveles } \\
\text { de hematocrito, } \\
\text { hemoglobina y } \\
\text { Glóbulos } \\
\text { blancos, } \\
\text { volumen } \\
\text { líquidos de } \\
\text { intravenoso } \\
\text { intraoperatorio, } \\
\text { Abuso de alcohol } \\
\text { y tabaco. }\end{array}$ & $\begin{array}{l}\text { Edad } \\
\text { Avanzada } \\
\text { No estar } \\
\text { casado } \\
\text { Hombre } \\
\text { Admisión } \\
\text { temprana } \\
\text { Estancia en } \\
\text { UCI }^{\dagger} \text { durante } \\
\text { una noche. }\end{array}$ \\
\hline $\begin{array}{l}\text { Patti R } \\
\text { et al. } \\
2011\end{array}$ & $\begin{array}{l}\text { N=100 } \\
\text { pacientes } \\
\text { carcinoma } \\
\text { colorrectal. } \\
\text { >65 años } \\
\text { OBSERVACIO } \\
\text { NAL } \\
\text { PROSPECTIVO }\end{array}$ & $\begin{array}{l}\text { CIRUGÍA } \\
\text { GASTRO- } \\
\text { INTESTINAL } \\
\text { COLORREC } \\
\text { TAL } \\
\text { DPO : } 18 \%\end{array}$ & $\begin{array}{l}\text { Edad, Historia } \\
\text { previas de DPO*, } \\
\text { abuso de alcohol, } \\
\text { Hipoalbuminemia } \\
\text { hipotensión } \\
\text { intraoperatoria, } \\
\text { volumen de } \\
\text { infusión elevado } \\
\text { y perdida } \\
\text { excesiva de } \\
\text { sangre. }\end{array}$ & $\begin{array}{l}\text { Hipoalbumine } \\
\text { mia } \\
\text { Abuso de } \\
\text { alcohol } \\
\text { Hipotensión } \\
\text { intraoperatorio }\end{array}$ \\
\hline $\begin{array}{l}\text { Ansalo } \\
\text { ni L et } \\
\text { al. }{ }^{57} \\
2010\end{array}$ & $\begin{array}{l}\text { N=351 } \\
\text { pacientes } \\
\text { cirugía general. } \\
\text { >65 años } \\
\text { OBSERVACIO } \\
\text { NAL } \\
\text { ANALíTICO } \\
\text { CASO- } \\
\text { CONTROL }\end{array}$ & $\begin{array}{l}\text { CIRUGÍA } \\
\text { GENERAL } \\
\text { URGENTE Y } \\
\text { ELECTIVA } \\
\text { DPO :13,2\% }\end{array}$ & $\begin{array}{l}\text { Edad avanzada } \\
\text { (>75 años). Co- } \\
\text { Morbilidad, } \\
\text { deterioro } \\
\text { cognitivo } \\
\text { preoperatorio, } \\
\text { síntomas psicopatológicos, } \\
\text { psicontroles de }\end{array}$ & $N / e^{\ddagger}$ \\
\hline
\end{tabular}




\begin{tabular}{|c|c|c|c|c|}
\hline & & & $\begin{array}{l}\text { glucemias } \\
\text { anormales }\end{array}$ & \\
\hline $\begin{array}{l}\text { Tei M. M. } \\
\text { et al. } \\
2010 \\
201\end{array}$ & $\begin{array}{l}\mathbf{N}=129 \\
\text { pacientes } \\
\text { carcinoma } \\
\text { colorrectal. } \\
\text { >71años } \\
\text { OBSERVACIO } \\
\text { NAL } \\
\text { RETROSPECTI } \\
\text { VO }\end{array}$ & $\begin{array}{l}\text { CIRUGÍA } \\
\text { GASTRO- } \\
\text { INTESTINAL } \\
\text { COLORREC } \\
\text { TAL } \\
\text { DPO :10,9\% }\end{array}$ & $\begin{array}{l}\text { Edad Avanzada } \\
\text { ASA }^{\S} \text { elevada } \\
\text { Encefalopatía } \\
\text { Estado } \\
\text { Nutricional (PNI) }\end{array}$ & $\begin{array}{l}\text { Encefalopatía } \\
\text { Estado } \\
\text { Nutricional } \\
(\text { PNI) }\end{array}$ \\
\hline $\begin{array}{l}\text { Morimo } \\
\text { to Y et } \\
\text { al. }{ }^{55} \\
2009\end{array}$ & $\begin{array}{l}\mathbf{N}=20 \text { pacientes } \\
\text { con cirugía } \\
\text { abdominal. } \\
>65 \text { años } \\
\text { OBSERVACIO } \\
\text { NAL } \\
\text { PROSPECTIVO }\end{array}$ & 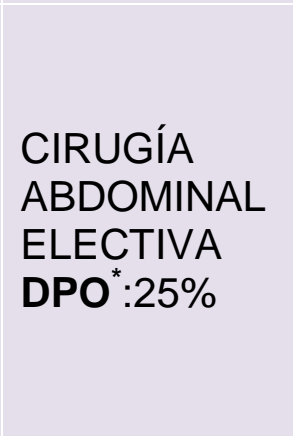 & $\begin{array}{l}\text { Edad Avanzada, } \\
\text { función cognitiva } \\
\text { baja, Saturación } \\
\text { de Oxigeno } \\
\text { cerebral } \\
\text { disminuida y el } \\
\text { retraso en la } \\
\text { recuperación de } \\
\text { la anestesia. }\end{array}$ & $\begin{array}{l}\text { Edad } \\
\text { Avanzada } \\
\text { La función } \\
\text { cognitiva baja } \\
\text { La Saturación } \\
\text { de Oxigeno } \\
\text { cerebral } \\
\text { disminuida }\end{array}$ \\
\hline $\begin{array}{l}\text { Koebru } \\
\text { gge B. } \\
\text { et al. }{ }^{54} \\
2009\end{array}$ & $\begin{array}{l}\text { N=71 pacientes } \\
\text { cirugía } \\
\text { abdominal } \\
\text { electiva } \\
>65 \text { años } \\
\text { OBSERVACIO } \\
\text { NAL } \\
\text { DESCRIPTIVO } \\
\text { PROSPECTIVO }\end{array}$ & $\begin{array}{l}\text { CIRUGÍA } \\
\text { ABDOMINAL } \\
\text { ELECTIVA } \\
\text { DPO }^{*}: 24 \%\end{array}$ & $\begin{array}{l}\text { Edad Avanzada } \\
\text { (>74 años), } \\
\text { estancias } \\
\text { prolongadas en } \\
\text { UCI }{ }^{\dagger}, \\
\text { hospitalaria, y } \\
\text { mayor número de } \\
\text { complicaciones } \\
\text { postoperatorias, } \\
\text { bajas puntuación } \\
\text { cognitivas y ASA } \\
\text { elevado. }\end{array}$ & $\begin{array}{l}\text { Edad > } 74 \\
\text { años }\end{array}$ \\
\hline $\begin{array}{l}\text { Ganai } \\
\text { S. et } \\
\text { al. }{ }^{53} \\
2007\end{array}$ & $\begin{array}{l}\text { N=228 } \\
\text { pacientes } \\
\text { Cirugía } \\
\text { abdominal. } \\
\text { N=89 Pacientes } \\
\text { con alto riesgo } \\
\text { de Delirium } \\
>70 \text { años } \\
\text { OBSERVACIO } \\
\text { NAL } \\
\text { SERIE DE } \\
\text { CASOS } \\
\text { RETROSPECTI } \\
\text { VO }\end{array}$ & $\begin{array}{l}\text { CIRUGÍA } \\
\text { ABDOMINAL } \\
\text { ELECTIVA Y } \\
\text { URGENCIA } \\
\text { DPO*:60\% }\end{array}$ & $\begin{array}{l}\text { Edad Avanzada } \\
\text { (79-82 años), } \\
\text { morbilidad, } \\
\text { restricción física, } \\
\text { cateterismo } \\
\text { vesical, } \\
\text { administración de } \\
\text { más de cuatro } \\
\text { medicamentos } \\
\text { nuevos } \\
\text { precipitantes, } \\
\text { reposo en cama } \\
\text { prolongado, } \\
\text { estado pobre en } \\
\text { fluidos, mal mal mal } \\
\text { control } \\
\text { glucémico, } \\
\text { hipoxia, anemia, } \\
\text { dolor nor no }\end{array}$ & $\begin{array}{l}\text { Estado } \\
\text { Nutricional } \\
\text { Precario. } \\
\text { Deterioro } \\
\text { Funcional. }\end{array}$ \\
\hline
\end{tabular}




$$
\begin{aligned}
& \text { controlado, } \\
& \text { trastornos } \\
& \text { metabólicos, } \\
& \text { hipoalbuminemia, } \\
& \text { malnutrición y } \\
& \text { caídas }
\end{aligned}
$$

"DPO: Delirium postoperatorio. Incidencia; ${ }^{\dagger} \mathrm{UCl}$ : Unidad de cuidados intensivos; ${ }^{\ddagger} \mathbf{N} / \mathbf{e}$ : No se especifica en el resumen del trabajo. Articulo de no libre difusión; ${ }^{\S}$ ASA: Sociedad Americana de Anestesiólogos; ;*PNI: índice de Pronóstico nutricional.

\section{DISCUSIÓN}

La edad avanzada ${ }^{53-59}$ sigue siendo el principal factor de riesgo predisponente y predictor independiente de riesgo asociado al delirium postoperatorio en cirugía general en los estudios actuales revisados, lo cual se ajusta a los hallazgos en otros estudios con diferentes pacientes quirúrgicos y médicos. ${ }^{9,46,60-62}$. Esto puede ser explicado por el deterioro de las funciones biológicas y capacidad funcional que conlleva el envejecimiento en relación con el del acto quirúrgico.

La morbilidad, el deterioro cognitivo, antecedentes previos de delirium, psicopatologías y las limitaciones físicas son factores de riesgo predisponentes asociados al DPO en ancianos intervenidos de cirugía General ${ }^{53-55,57-59}$. El ser hombre y soltero fue estudiado como factor de riesgo y predictor independiente de DPO, por Mangnall LT y colaboradores ${ }^{59}$ lo que se confirma en otros estudios ${ }^{63-64}$. Los autores justifican esta asociación debida a un déficit general relacionada con el sexo y el comportamiento saludable, al igual que la relevancia desempeñada por la familia en este proceso puede contribuir a la mejora del delirum sobre aquellos pacientes que no tienen ese apoyo.

Los factores de riesgo modificables ${ }^{53-56,58-59}$ que precipitan este síndrome en este subgrupo de pacientes quirúrgicos y que lo hacen característico en relación a los pacientes médicos han sido el tiempo de ingreso en relación con el procedimiento quirúrgico, la duración de la cirugía, la estancia prolongada en $\mathrm{UCI}$, la hipotensión intraoperatoria, el volumen de infusión de líquidos elevados, la puntuación ASA alta, saturación de oxígeno cerebral disminuida, dolor postoperatorio mal controlado, retraso en la recuperación de la anestesia y mayor número de complicaciones postoperatorias (Tabla II). Esta diferencia entre los factores de riesgo del paciente quirúrgico y el médico ya fue descrita en la literatura médica por otros autores ${ }^{9}$.

Aunque existen muchos más factores de riesgos relacionados con el DPO en esta revisión sólo hemos mostrado los estudiados por algunos autores y limitados a una población quirúrgica determinada (Tabla II). Se sigue confirmando la etiología multifactorial del delirium y su alta tasa de incidencia ${ }^{53-59}$. 


\section{TABLA II. Factores de Riesgo predisponentes y precipitantes del delirium postoperatorio en Ancianos tras cirugía General.}

\begin{tabular}{|c|c|c|c|c|c|c|}
\hline Mangnall LT et al ${ }^{59}$ & $\begin{array}{l}\text { Patti R } \\
\text { et al. }\end{array}$ & $\begin{array}{c}\text { Ansaloni } L \text { et } \\
\text { al. }^{57}\end{array}$ & $\begin{array}{c}\text { Tei M. et } \\
\text { al. }^{56}\end{array}$ & $\begin{array}{l}\text { Morimoto } \\
\text { Y et al. }{ }^{55}\end{array}$ & $\begin{array}{c}\text { Koebrugge B. } \\
\text { et al. }{ }^{54}\end{array}$ & $\begin{array}{l}\text { Ganai S. } \\
\text { et al. }{ }^{53}\end{array}$ \\
\hline \multicolumn{7}{|c|}{ FACTORES PREDISPONENTES (NO MODIFICABLE) } \\
\hline $\begin{array}{l}\text { Edad avanzada } \\
\text { Hombre } \\
\text { Morbilidades } \\
\text { Limitaciones físicas }\end{array}$ & $\begin{array}{l}\text { Edad Avanzada } \\
\text { Antecedentes de } \\
\text { delirium }\end{array}$ & $\begin{array}{l}\text { Edad } \\
\text { avanzada } \\
\text { (>75 años) } \\
\text { Morbilidad } \\
\text { Psicopatología } \\
\text { Deterioro } \\
\text { cognitivo }\end{array}$ & $\begin{array}{l}\text { Edad } \\
\text { Avanzada }\end{array}$ & $\begin{array}{l}\text { Edad } \\
\text { Avanzada. } \\
\text { Función } \\
\text { cognitiva } \\
\text { baja }\end{array}$ & $\begin{array}{l}\text { Edad } \\
\text { Avanzada } \\
\text { (>74 años) } \\
\text { Baja } \\
\text { puntuación } \\
\text { cognitivas }\end{array}$ & $\begin{array}{l}\text { Edad Avanzada } \\
\text { ( } 79-82 \text { años) } \\
\text { Morbilidad }\end{array}$ \\
\hline \multicolumn{7}{|c|}{ FACTORES PRECIPITANTES (MODIFICABLES) } \\
\hline $\begin{array}{l}\text { Soltero } \\
\text { Estancia en UCI* } \\
\text { El tiempo de ingreso } \\
\text { hospitalario en relación con } \\
\text { el procedimiento quirúrgico } \\
\text { Duración de la cirugía } \\
\text { Niveles de hematocrito, } \\
\text { hemoglobina y Glóbulos } \\
\text { blancos } \\
\text { Infusión intravenosa } \\
\text { intraoperatoria } \\
\text { Consumo de alcohol } \\
\text { Consumo de tabaco. }\end{array}$ & $\begin{array}{l}\text { Abuso de alcohol } \\
\text { Hipoalbuminemia. } \\
\text { Hipotensión } \\
\text { intraoperatoria } \\
\text { Volumen de } \\
\text { infusión elevado } \\
\text { Perdida excesiva } \\
\text { de sangre. }\end{array}$ & $\begin{array}{l}\text { Mal control } \\
\text { glucémico }\end{array}$ & $\begin{array}{l}\text { ASA elevada } \\
\text { Encefalopatía } \\
\text { Estado } \\
\text { Nutricional } \\
\text { (PNI†) }\end{array}$ & $\begin{array}{l}\text { Saturación } \\
\text { de Oxigeno } \\
\text { cerebral } \\
\text { disminuida } \\
\text { Retraso en } \\
\text { la } \\
\text { recuperación } \\
\text { de la } \\
\text { anestesia. }\end{array}$ & $\begin{array}{l}\text { Estancias } \\
\text { prolongadas en } \\
\text { UCI* } \\
\text { Estancia } \\
\text { hospitalaria } \\
\text { más } \\
\text { prolongada } \\
\text { Mayor } \text { n }^{\circ} \text { de } \\
\text { complicaciones } \\
\text { postoperatorias } \\
\text { ASAł } \\
\text { elevado. }\end{array}$ & $\begin{array}{l}\text { La restricción } \\
\text { física } \\
\text { Cateterismo } \\
\text { vesical } \\
\text { Adm. + } 4 \\
\text { medicamentos } \\
\text { nuevos o } \\
\text { precipitantes } \\
\text { Reposo en cama } \\
\text { prolongado } \\
\text { Estado pobre en } \\
\text { fluidos } \\
\text { Mal control } \\
\text { glucémico } \\
\text { Hipoxia } \\
\text { Anemia } \\
\text { Dolor no } \\
\text { controlado } \\
\text { Trastornos } \\
\text { metabólicos } \\
\text { Hipoalbuminemia } \\
\text { Malnutrición } \\
\text { Caídas }\end{array}$ \\
\hline
\end{tabular}

*UCI: Unidad de cuidados intensivos; †PNI: índice de Pronóstico nutricional. †ASA: Sociedad Americana de Anestesiólogos;

Los predictores de riesgo a diferencia de los factores de riesgo nos explican la probabilidad de que acontezca el delirium y no solo describen las circunstancias que condicionan el delirium, lo cual, permite identificar a los grupos de riesgo y evitar la aparición del mismo. En los estudios examinados podemos ver que no existe una homogeneidad en los factores de predicción (Taba I), lo mismo que sucede en otras poblaciones de pacientes quirúrgicos ${ }^{37,60,61,65}$, lo que hace más difícil generar modelos de predicción unificados.

La prevención y el tratamiento de delirium deben considerarse como una prioridad en nuestro contexto hospitalario. Aplicándole la importancia y urgencia que merece.

Existen diferentes estudios, desde hace mas de 40 años, que analizan y respaldan, la eficacia de llevar a cabo diferentes estrategias especificas de intervención no farmacológicas (control de factores de riesgo modificables) y su efecto positivo sobre la disminución de la incidencia del delirium hospitalario en distintos colectivos de pacientes ancianos ${ }^{11-17,66}$ sin olvidarnos de la importancia que conlleva una adhesión adecuada, para la optimización de los resultados, en las intervenciones no farmacológicas. ${ }^{67}$

Cuando las estrategias de prevención están orientadas con intervenciones multifactorial y llevadas a fin por un equipo interdisciplinario especializado en la materia, gozan de la combinación potencial ideal para reducir el delirium. ${ }^{8}$ 
Esta revisión justificaría una nueva línea de investigación, cuya meta sería elaborar un estudio de intervención a partir de la realización de un protocolo, con directrices no farmacológicas, basándonos en los factores de riesgos postoperatorios reflejados en este trabajo y evaluar su eficacia en pacientes quirúrgicos ancianos candidatos a cirugía general.

\section{CONCLUSIÓN}

Al DPO se le debe de prestar más atención porque es un indicador de calidad hospitalario que genera elevado coste económico y humano, además de ser un síndrome grave, frecuente, predecible, prevenible, reversible, que suele estar mal diagnosticado y tratado.

Son pocos los estudios observacionales que estudian los factores de riesgo en la cirugía general, mayoritariamente su población a estudio son los pacientes de cirugía cardiaca y ortopédica, en concreto las fracturas de cadera.

En la gran mayoría de los estudios revisados los grupos los clasificaban en pacientes cardiacos o no cardiacos, incrementando la dificultad en la selección de los trabajos por la heterogeneidad de la muestra.

Existe gran disparidad de predictores de riesgo quirúrgico, solo la edad coincide en todos ellos.

Los factores de riesgos predisponentes y precipitantes de DPO son de naturaleza multifactorial en este subgrupo de población quirúrgica.

El tiempo de ingreso en relación con el procedimiento quirúrgico, la duración de la cirugía, la estancia prolongada en UCI, la hipotensión intraoperatoria, el volumen de infusión de líquidos elevados, la puntuación ASA alta, saturación de oxigeno cerebral disminuida, dolor postoperatorio mal controlado, retraso en la recuperación de la anestesia y mayor número de complicaciones postoperatorias son factores de riesgo modificables propios del ancianos con cirugía general, derivados del acto quirúrgico.

La identificación metodología de pacientes en riesgo es fundamental para la prevención, la detección temprana y el tratamiento adecuado del delirium, primordialmente porque indica la presencia de una alteración orgánica subyacente que hay que resolver.

\section{BIBLIOGRAFÍA}

1. Etzioni DA, Liu JH, Maggard MA, Ko CY. The aging population and its impact on the surgery workforce. Ann Surg. Ann Surg. 2003; 238:170-7.

2. Fuentes Valdés Edelberto, Jiménez Paneque Rosa. Riesgo quirúrgico en pacientes mayores de 60 años. Rev Cubana cir [revista en la Internet]. 2000 Abr [citado 2012 Dic 18] ; 39(1): 73-81. Disponible en: http://scielo.sld.cu/scielo.php?script=sci arttext\&pid=S003474932000000100011\&lng=es.

3. Audissio RA, Veronesi P, Ferrani L, Cipolla C, Andreoni B, Aapro W. Elective surgery for gastrointestinal tumors en the elderly. Ann Oncol.1997;8(4):317-26. 
4. Corujo E, Pérez DG. Cambios más relevantes y peculiaridades de las enfermedades en el anciano. En: Sociedad Española de Geriatría y Gerontología. Tratado de Geriatría para Residentes. Madrid. SEGG; 2007. pag 47-58.

5. Rudolph JL, Marcantonio ER. Review articles: postoperative delirium: acute change with long-term implications. Anesth Analg. 2011;112(5):1202-11

6. Noimark D. Predicting the onset of delirium in the post-operative patient. Age Ageing. 2009; 38(4):368-73.

7. Barbosa Fabiano Timbó, Cunha Rafael Martins da, Pinto André Luiz Carvalho Leme Teixeira. Delirium pós-operatório em idosos. Rev Bras Anestesiol. [revista en la Internet]. 2008 Dic [citado 2012 Dic 18]; 58(6): 665-670. Disponible en: http://www.scielo.br/scielo.php?script=sci arttext\&pid=S0034-

70942008000600012\&lng=es. $\quad$ http://dx.doi.org/10.1590/S003470942008000600012 .

8. Tropea J, Slee JA, Brand CA, Gray L, Snell T.Clinical practice guidelines for the management of delirium in older people in Australia.Australas $\mathrm{J}$ Ageing. 2008;27(3):150-6.

9. Inouye SK. Delirium in older persons. N Engl J Med. 2006;354:1157-65

10. Rasmussen LS, O’Brien JT, Siverstein JH et al. Is perioperative cortisol secretion related to postoperative cognitive dysfunction?. Acta Anaesthesiol Scand. 2005;49:1225-31.

11. Lazarus HR, Hagens JH. Prevention of psychosis following open-heart surgery. Am J Psychiatry. 1968; 124: 1190-5.

12. Budd S, Brown W. Effect of a reorientation technique on postcardiotomy delirium. Nurs Res. 1974;23(4):341-8.

13. Owens JF, Hutelmyer CM. The effect of postoperative intervention on delirium in cardiac surgical patients. Nurs Res. 1982;31(1):60-2.

14. Björkelund KB, Hommel A, Thorngren KG, Gustafson L, Larsson S, Lundberg D. Reducing delirium in elderly patients with hip fracture: a multi-factorial intervention study. Acta Anaesthesiol Scand. 2010;54(6):678-88.

15. Martinez FT, Tobar C, Beddings Cl, Vallejo G, Fuentes P. Preventing delirium in an acute hospital using a non-pharmacological intervention. Age Ageing. 2012;41(5):629-34.

16. Greer N, Rossom R, Anderson P, MacDonald R, Tacklind J, Rutks I, et al. Delirium: Screening, Prevention, and Diagnosis - A Systematic Review of the Evidence [Internet]. Washington (DC): Department of Veterans Affairs (US); 2011 Sep. Available from http://www.ncbi.nlm.nih.gov/books/NBK82554/ PubMed PMID: 22206108.

17. Tabet N, Howard R. Non-pharmacological interventions in the prevention of delirium. Age Ageing. 2009;38(4):374-9.

18. Leslie DL, Inouye SK. The importance of delirium: economic and societal costs. J Am Geriatr Soc. 2011;59(2):S241-3.

19. Smith DJ. Intervención Enfermera Cuidado postoperatorio. En: Lewis SM, McLean M, Dirksen SR, O’brien, P. et al. Enfermería médico quirúrgica. $6^{a}$ ed. Madrid: Mosby; 2004. Pg 405-26

20. Carrillo-Esper R, Medrano-del Ángel T. Delirium y disfunción cognitiva postoperatorios. Rev Mex Anest. 2011; 34(3): 211-19

21. Newman S, Stygall J, Hirani S, Shaefi S, Maze M. Postoperative cognitive dysfunction after noncardiac surgery: a systematic review. Anesthesiology. 2007;106:572-90

22. Delirium, demencia, trastornos amnésicos y otros trastornos cognoscitivos. In: López-Ibor Aliño JJ, Valdés Miyar M, editors. DSM-IV-TR. Manual diagnóstico y estadístico de los trastornos mentales: texto revisado. Barcelona: Masson; 2005. 
23. Lipowski ZJ. Delirium: How its concept has developed. Int Psychogeriatr. $1991 ; 3(2): 115-20$

24. Lipowski ZJ. Delirium (acute confusional states). JAMA.1987; 258:1789-92.

25. Bekker AY, Weeks EJ. Cognitive function after anesthesia in the elderly. Best Pract Res Clin Anaesthesiol.2003; 17:259-72

26. Kain ZN, Caldwell-Andrews AA, Maranets I, McClain B, Gaal D, Mayes LC, et al. Preoperative anxiety and emergence delirium and postoperative maladaptive behaviors. Anesth Analg. 2004; 99: 1648-54.

27. Laurila JV, Pitkala KH, Strandberg TE, Tilvis RS. The impact of different diagnostic criteria on prevalence rates for delirium. Dement Geriatr Cognit Disord. 2003;16(3):156-61

28. Voyer P, Richard S, Doucet, L, Carmichael PH. Detecting delirium and subsyndromal delirium using different diagnostic criteria among demented longterm care residents. J Am Med Dir Assoc. 2009; 10(3):181-8

29. Engelhard K, Werner C. Postoperative cognitive dysfunction in geriatric patients. Anasthesiol Intensivmed Notfallmed Schmerzther. 2008; 43(9):606-14.

30. Stern Y. What is cognitive reserve? Theory and research application of the reserve concept. J Int Neuropsychol Soc .2002; 8(3):448-60.

31. Tejeiro Martínez J, Gómez Sereno B. Guía diagnóstica y terapéutica del síndrome confusional agudo. Rev Clin Esp. 2002; 202 (5):280-288

32. Duppils GS, WikBlad K. Delirium: behavioural changes before and during the prodromal phase. J Clin Nurs. 2004:13(5);609-16

33. Leslie DL, Marcantonio ER, Zhang Y, Leo-Summers L, Inouye SK. One-year health care costs associated with delirium in the elderly population. Arch Intern Med.2008: 14; 168(1):27-32.

34. Muñoz Rodríguez PE. Test de Cribado en la práctica clínica. En: Bulbena Vilarrasa A, Berrios E G, Fernández de Larrinoa Palacios P, editores. Medición clínica en psiquiatría y psicología. Barcelona: Masson; 2000. p. 35-51

35. González M, de Pablo J, Fuente E, Valdés M, Peri JM, Nomdedeu M, et al. Instrument for detection of delirium in general hospitals: adaptation of the confusion assessment method. Psychosomatics. 2004; 45(5):426-31.

36. Téllez JM, Villena A, Morena S, Pascual P, López C. El paciente agitado. Guías Clínicas. 2005;5(34).Disponible

en: http://www.uclm.net/profesorado/jtorre/DOCUMENTOS/PSIQUIATR/temas/tema1/ Agitado.pdf

37. Martínez-Velilla N, Alonso Bouzón C, Ripa Zazpe C, Sánchez-Ostiz R. Acute postoperative confusional syndrome in the elderly patient. Cir Esp. 2012; 90(2):7584.

38. Agency for Healthcare Research and Quality (AHRQ). National Quality Measures Clearinghouse ( NQHC) [base de datos internet].EEUU: AHRQ;1997, [Dic. 2012; citado el 10 dic. 2012]. Disponible en: http://www.qualitymeasures.ahrq.gov/index.aspx

39. Cole MG. Delirium in elderly patients. Am J Geriatr Psychiatry.2004;12(1):7-21

40. O’keeffe ST, Ní Chonchubhair A. Postoperative delirium in the elderly. $\mathrm{Br} \mathrm{J}$ Anaesth. 1994; 73(5): 673-87.

41. Cofer MJ. Un compañero inoportuno del anciano: el delirio postoperatorio. Nursing. 2006; 24(1):21-23.

42. Robinson TN, Raeburn CD, Tran ZV, Brenner LA, Moss M. Motor subtypes of postoperative delirium in older adults. Arch Surg. 2011;146(3):295-300.

43. Katznelson R, Djaiani G, Tait G, Wasowicz M, Sutherland AM, Styra R, et al. Hospital administrative database underestimates delirium rate after cardiac surgery. Can J Anaesth. 2010;57(10):898-902. 
44. Marcantonio ER. Postoperative delirium: a 76-year-old woman with delirium following surgery. JAMA. 2012;308(1):73-81.

45. Britton A, Russell R. Multidisciplinary team interventions for delirium in patients with chronic cognitive impairment. Cochrane Database Syst Rev. 2004;(2):CD000395. PubMed PMID: 11279689.

46. Lipowski ZJ. Delirium in the elderly patient. N Engl J Med. 1989; 320(9): 578-82

47. Andrew MK, Freter SH, Rockwood K. Incomplete functional recovery after delirium in elderly people: a prospective cohort study. BMC Geriatrics.2005; 5:5

48. Moller JT, Cluitmans P, Rasmussen LS, et al. Long-term postoperative cognitive dysfunction in the elderly ISPOCD1 study. ISPOCD investigators. International Study of Post-Operative Cognitive Dysfunction. Lancet. 1998;351(9106):857-61

49. González Al. Estados confusionales agudos en el anciano: papel de la enfermería. Revista de Enfermería Gerontológica.2005; 10: 48-49.

50. Moreno A, Gallego R, Martín ME, Ballesteros E. Experiencias de las cuidadoras familiares e inmigrantes ante el delirium de los mayores en el hospital. ENE: Resvista de Enfermería. 2010; 4(2): 33-9.

51. Inouye SK, Bogardus ST, Charpentier PA, Leo-Summers L, Acampora D, Holford TR, et al. A multicomponent intervention to prevent delirium in hospitalized older patients. N Engl J Med.1999;340 (9):669-76.

52. Sykes PK. Prevention and management of postoperative delirium among older patients on an orthopedic surgical unit: a best practice implementation project. J Nurs Care Qual. 2012; 27(2):146-53.

53. Ganai S, et al. Adverse outcomes of geriatric patients undergoing abdominal surgery who are at high risk for delirium Arch Surg. 2007; 142(11):1072-8.

54. Koebrugge B, Koek HL, van Wensen RJ, Dautzenberg PL, Bosscha K. Delirium after abdominal surgery at a surgical ward with a high standard of delirium care: incidence, risk factors and outcomes. Dig Surg. 2009; 26(1):63-8.

55. Morimoto Y, Yoshimura M, Utada K, Setoyama K, Matsumoto M, Sakabe T. Prediction of postoperative delirium after abdominal surgery in the elderly. J Anesth. 2009; 23(1):51-6.

56. Tei M, Ikeda M, Haraguchi N, Takemasa I, Mizushima T, Ishii $\mathrm{H}$ et al. Risk factors for postoperative delirium in elderly patients with colorectal cancer. Surg Endosc. 2010; 24(9):2135-9.

57. Ansaloni L, Catena F, Chattat R, Fortuna D, Franceschi C, Mascitti P, Melotti RM. Risk factors and incidence of postoperative delirium in elderly patients after elective and emergency surgery. Br J Surg. 2010; 97(2):273-80.

58. Patti R, Saitta M, Cusumano G, Termine G, Di Vita G. Risk factors for postoperative delirium after colorectal surgery for carcinoma. Eur J Oncol Nurs. 2011; 15(5):519-23.

59. Mangnall LT, Gallagher R, Stein-Parbury J. Postoperative delirium after colorectal surgery in older patients. Am J Crit Care. 2011; 20(1):45-55.

60. Marcantonio ER, Goldman L, Mangione CM, Ludwig LE, Muraca B, Haslauer $\mathrm{CM}$, et al. A clinical prediction rule for delirium after elective noncardiac surgery. JAMA. 1994;271:134-9.

61. Freter SH, Dunbar MJ, MacLeod H, Morrison M, MacKnight C, Rockwood K. Predicting post-operative delirium in elective orthopaedic patients: the Delirium Elderly At-Risk (DEAR) instrument. Age Ageing. 2005;34:169-71.

62. Litaker D, Locala J, Franco K, Bronson DL, and Tannous Z, Preoperative risk factors for postoperative delirium. General Hospital Psychiatry, 2001. 23(2):84-9 
63. Edelstein DM, Aharonoff GB, Karp A, Capla EL, Zuckerman JD, Koval KJ.Effect of postoperative delirium on outcome after hip fracture. Clin Orthop Relat Res. 2004;(422):195-200.

64. Edlund A, Lundstrom M, Brannstrom B, Bucht G, Gustafson Y. Delirium before and after operation for femoral neck fracture. J Am Geriatr Soc.2001;49:1335-1340.

65. Rudolph JL, Jones RN, Levkoff SE, Rockett C, Inouye SK, Sellke FW, et al. Derivation and validation of a preoperative prediction rule for delirium after cardiac surgery. Circulation. 2009;119: 229-36.

66. Carrera C. Rol de enfermería en la prevención del delirium en ancianos hospitalizados con fractura de cadera. Recomendaciones Generales. Enferm Global. 2012; 11(3):356-78.

67. Inouye SK, Bogardus ST Jr, Williams CS, Leo-Summers L, Agostini JV. The role of adherence on the effectiveness of non pharmacologic interventions: evidence from the delirium prevention trial. Arch Intern Med. 2003;163(8):958-64. 